\title{
(อ) OPEN ACCESS \\ Concussion and long-term cognitive impairment among professional or elite sport-persons: a systematic review
}

\author{
Valentina Gallo (1) , 1,2,3 Kim Motley, ${ }^{4}$ Simon P T Kemp, ${ }^{3,5}$ Saba Mian, ${ }^{3}$ Tara Patel, ${ }^{1,6}$ \\ Laura James, ${ }^{1}$ Neil Pearce, ${ }^{3}$ Damien McElvenny ${ }^{3,7,8}$
}

- Additional material is published online only. To view please visit the journal online (http://dx.doi.org/10.1136/ innp-2019-321170).

${ }^{1}$ Institute of Population Health Sciences, Queen Mary, University of London, London, UK

${ }^{2}$ School of Public Health, Imperial College London, London, UK

${ }^{3}$ Department of Medical Statistics, London School of Hygiene and Tropical, London, UK

${ }^{4} S$ chool of Medicine and Dentistry, Queen Mary University of London, London, UK

${ }^{5}$ Rugby Football Union, Twickenham, London, UK ${ }^{6} \mathrm{BSC}$ in Biology, Imperial College London, London, UK ${ }^{7}$ Institute for Occupational Medicine Edingburgh, Edingburgh, UK

${ }^{8}$ University of Manchester, Manchester, UK

\section{Correspondence to}

Dr Valentina Gallo, CPCPH, Barts and The London School of Medicine and Dentistry, London E1 2AB, UK; v.gallo@qmul.ac.uk

VG and KM contributed equally.

Received 10 May 2019 Revised 20 December 2019 Accepted 26 January 2020 Published Online First 27 February 2020

\section{ABSTRACT}

Introduction Understanding whether concussion in sport is associated with worsening cognitive function in later life will likely have immediate repercussion on sports concussion prevention and management policy and sporting rules and regulations. This systematic review aims to summarise the evidence on the association between concussion sustained by professional/elite athletes and long-term cognitive impairment.

Methods Embase, PubMed and Web of Science were used to search for eligible studies. Studies including professional/elite athletes from any sport were considered. Three comparison groups were considered: internal comparison (concussed vs non-concussed athletes within the same sample); between-sport comparison (contact sport athletes vs non-contact sports ones); external comparison (athletes vs samples of the general population or population norms)

Results 14 studies were included (rugby, American football, ice hockey players, boxers and marital art fighters). The general quality of the evidence was poor. The overall evidence, weighted for type of comparison and study quality, points towards an association between sustaining a sport-related concussion and poorer cognitive function later in life in rugby, American football and boxing, although it is unclear to what extent this is clinically relevant. Data on ice hockey and martial arts were too sparse to allow conclusions to be drawn.

Conclusion High-quality, appropriately designed and powered epidemiological studies are urgently needed to assess the association between sustaining a sportrelated concussion and cognitive impairment later in life. Particular emphasis should be put on the clinical translational value of findings.

\section{INTRODUCTION}

Since Martland, in $1928,{ }^{1}$ first described the clinical syndrome of 'dementia puglistica' in boxers presenting with confusion, slowed movement and parkinsonian symptoms following repeated blows to the head, our understanding of the association between concussion and dementia in boxers has advanced considerably. ${ }^{2}$ Neuropathological features of dementia pugilistica, identified by Corsellis et al in $1973,{ }^{3}$ showed a consistent pattern of neuropathological changes in the post-mortem examination of the brains of retired boxers. Since then, further investigations into the mechanisms that may underlie these changes, in both boxing and other contact sports have been conducted. ${ }^{4}$

More recently, research has suggested an association between traumatic brain injury and neurodegenerative conditions. ${ }^{5-9}$ Specifically, chronic traumatic encephalopathy (CTE) encompasses a clinical spectrum of motor, psychological and cognitive symptoms and is a progressive neurodegenerative condition thought to be caused by single or repetitive concussion-related trauma. ${ }^{4} 10$ The clinical features of CTE show some resemblance to the progressive cognitive decline and neuropsychiatric presentation associated with Alzheimer disease,${ }^{11}$ including an insidious onset with amnestic mild cognitive impairment, and similar hallmark pathological features. ${ }^{1112}$ However, CTE has been described as a separate condition, ${ }^{13}$ although the pathology is potentially overlapping with that of Alzheimer diseases in up to $25 \%$ of the cases. ${ }^{14}$

The theoretical and operational definitions of sport-related concussion are a matter of ongoing debate, although they are all consistent in suggesting that it should be regarded as a mild traumatic brain injury. The latest 2016 Berlin Consensus Statement on Concussion in Sport concluded that 'concussion is a traumatic brain injury which (1) might be caused by a direct or indirect blow to the head; (2) typically results in the rapid onset of short-lived impairment of neurological function that resolves spontaneously; (3) may result in neuropathological changes but the acute clinical signs and symptoms largely reflect a functional disturbance rather than a structural injury and, as such, no abnormality is seen on standard structural neuroimaging studies and (4) might or might not involve loss of consciousness'. ${ }^{15}$ According to this definition, it has been shown that numerous athletes have been exposed to head injury events resulting in concussion during playing careers ${ }^{16}$; however, it is reasonable to assume that many more have been exposed to repetitive subconcussive head impact events (eg, when heading a football), the majority of which were below the threshold for a clinical diagnosis of concussion, depending on the nature of the often short-lived neurological symptoms and their interpretationparticularly a few decades ago, when there was less awareness about sport-related concussion.

While there is now a stronger understanding of the potential mechanisms involved in the processes underlying concussion, the epidemiological 
evidence and the strength of this evidence, to support the longterm effects on cognition remains unclear. ${ }^{17} \mathrm{~A}$ recent systematic review aimed at assessing the long-term neurological sequelae of sport-related concussion concluded that there might be an association with repeated concussion and later cognitive impairment. ${ }^{18}$ However, this review included also varsity and amateur athletes, and did not provide an in-depth analysis of the limitation of study design and potential for bias and confounding of the included papers. Understanding whether concussion in sport is significantly associated with worsening of cognitive function in later life is of great importance. Uncovering this possible association would likely have immediate repercussions on current concussion prevention and management policy, sports rules and regulations, and possibly on the listing of cognitive impairment as an occupational diseases for former professional sportspersons.

This systematic review aims to assess and summarise the evidence on the association between concussion sustained by professional/elite athletes and long-term cognitive function as assessed on neurocognitive testing or by clinical diagnosis. Given the recent changes of definitions of concussion, this is considered in broader term, but results are described by the adopted definition and its consistency with the latest consensus. ${ }^{15}$

\section{METHODS}

A review protocol was written up and agreed on by two of the co-authors (KM and VG), before the review started. It is available on request.

\section{Search strategy and terms}

Three databases, Embase, PubMed and Web of Science, were used to search for eligible studies. The key search terms 'sport (football, rugby, boxing, wrestling, ice hockey), athlete, concussion, traumatic brain injury, Alzheimer disease, dementia, MCI' were included in the database search. Prior to conducting each search, search terms were tested for suitability to maximise the focus of results relative to the search criteria outlined. Where appropriate, $\mathrm{MeSH}$ and Emtree indexing terms were utilised to broaden the coverage of the search. Full details of each search including the Population, Intervention, Control, and Outcome (PICO) criteria are included in online supplementary table 1. The search was conducted in February 2017 and updated in September 2018.

\section{Selection criteria and eligibility}

One reviewer screened the titles and abstracts of the output of the search to identify potentially eligible studies. Full texts for potentially eligible papers were obtained where possible, and independently assessed for eligibility by two reviewers. All included papers were additionally reviewed for references to other potentially relevant papers. To schematise the steps used for the selection of studies, a flowchart diagram was developed based on the PRISMA recommendations. ${ }^{19}$

\section{Inclusion criteria}

- Original, peer-reviewed articles

- Articles written in English

- Study designs: all designs were evaluated, including casecontrol studies, cohort studies, cross-sectional studies and case-series

- PICO criteria:

- Population-studies including professional or elite athletes, from any sport, with at least one season of competitive participation
- Exposure (intervention)-history of at least one sportrelated concussion. Any definition of concussion was considered in this review, however, the Berlin consensus definition ${ }^{15}$ (or analogous) was considered as the gold standard. Definition of concussion was extracted and noted, both clinically assessed or self-reported concussions were included. Repeated subconcussive head impacts were also considered for inclusion but results described separately for concussion and repeated subconcussive head impacts. Exposure to blow to the head resulting in concussion or repeated subconcussive head impact approximated by length of career of participating in sport or other suitable proxy measures, were included.

- Comparison group-three comparison groups were considered: (1) internal comparisons, when concussed athletes were compared with non-concussed athletes within the same sample; (2) between sport comparisons, when contact sport athletes were compared with non-contact sports athletes; (3) external comparisons, when athletes were compared with samples of the general population or population norms.

- Outcome-long-term cognitive function as assessed by neurocognitive tests or clinical evidence of mild cognitive impairment or Alzheimer disease or dementia assessed as clinical diagnosis (including self-reported doctordiagnosed), and/or additionally supported by cognitive testing. The methods used to assess cognitive impairment were recorded.

\section{Exclusion criteria}

- Single-case reports

- Research conducted on varsity athletes or high school sport participants

- Review articles and conference abstracts (but references were cross-checked to include any paper which might have been missed)

- Exposure to concussion in a setting different from professional or elite-level sport

- Acute rather than long-term effects of cognition investigated

- Neuropathological studies

When two or more papers reported results of a (partially) overlapping sample, the largest study was included. The PICO criteria, used to guide the inclusion and exclusion criteria, and to create the search criteria, are detailed in online supplementary table 1 .

\section{Data collection}

Each search was run individually and each result transferred to a separate file using a referencing programme (Endnote). Results from all three searches were then combined and any duplicates removed. An outline of this process is shown in figure 1 .

A spreadsheet for data extraction was created. Articles to be included in the review, were assessed and data extracted. Data including author, publication date, study design, participants and recruitment method were extracted in addition to any potential confounders for each study and source of funding. Outcome ascertainment and exposure assessment methods were extracted, with definitions, as applicable. Overall findings for each study plus any relevant subgroup analysis were also recorded.

Appraisal of study quality was conducted using the NewcastleOttawa scale. ${ }^{20}$ An adapted version of the scale was used for cross-sectional studies. This assessment tool aims to formulate a 


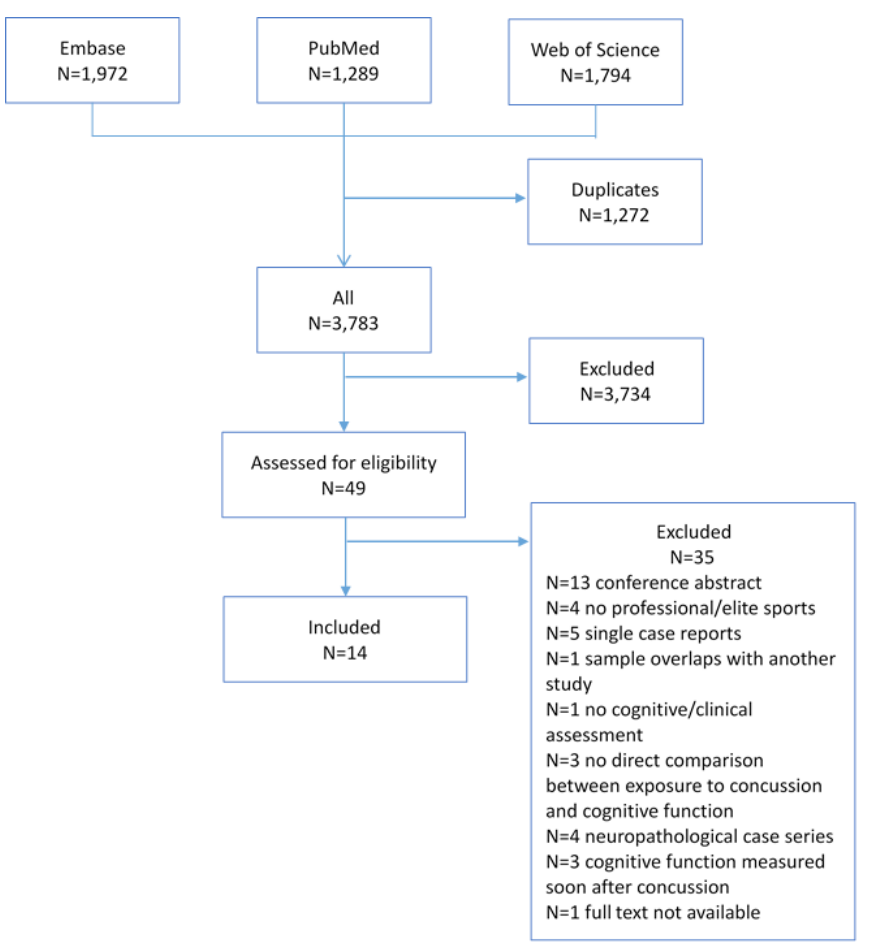

Figure 1 Flow chart of search process. Adapted from Moher et a/ ${ }^{19}$.

quality score for non-randomised studies included in a systematic review ${ }^{2021}$ (online supplementary table 2).

\section{RESULTS}

\section{Study selection}

Overall, 14 studies met the inclusion/exclusion criteria and were therefore included in the review ${ }^{22-35}$ (online supplementary table 3 ). A total of 35 studies were excluded because used a partially overlapping sample, did not include professional or elite-level athletes, were single case-reports, were based on neuropathological/brain bank series, were missing essential information on details of the cognitive assessment, there was no direct or suitably indirect comparison between concussion and cognition, cognitive assessment was measured immediately after concussion or because the full text was not available (figure 1).

\section{Study characteristics and design}

The data extracted are shown in tables $1-4$ and in online supplementary table 3. All studies, but one, had a cross-sectional design, with varying degree of representativeness of the source population, some with an external comparison group. Bang et al reported a case-series of five boxers. ${ }^{25}$ Nine studies compared different degrees of concussion within the same group of sportsmen (internal comparison) ${ }^{23} 26-33$; three studies also used as a comparison group another athlete population less exposed to concussion (between-sports comparison), ${ }^{24} 2731$ with the remaining using only non-athlete controls (external comparison). 22253435

Three studies included rugby players, ${ }^{27} 3132$ eight included American footballers, ${ }^{22-24} 2930$ 33-35 with two including ice hockey players, ${ }^{24} 28$ and two studies included boxers, ${ }^{25}{ }^{26}$ one of which compared boxers with martial arts fighters ${ }^{26}$ (online supplementary table 3 ).

Four studies did not disclose their source of funding 23262931 ; one study was funded by a sport federation, ${ }^{27}$ seven by academic/ governmental institutions 22242528303334 and one by a corporation for cognitive testing tools. ${ }^{35}$

\section{Quality of evidence}

The quality of the evidence was assessed against the most appropriate analysis assessing the effects of concussion, namely the internal comparison. Overall only two studies scored six or more on the Newcastle-Ottawa Scale. ${ }^{22} 26$ Three studies had what appeared to be representative samples ${ }^{27} 2932$ and only one study contained an a priori sample size calculation. ${ }^{26}$ None of the studies contained a description of the non-respondents. Four studies did not contain descriptions of exposure to concus$\operatorname{sion}^{24252735}$; and only half of the studies had an appropriate method of analysis for the internal comparison. $.^{22} 26-293233$ One study included both ice hockey players and American footballers making comparisons between each of these sport groups particularly difficult. ${ }^{24}$

\section{Exposure assessment}

Concussion was self-reported in all studies, unless it was estimated indirectly by the number of bouts fought, ${ }^{2526}$ or assumed to be high given the playing/fighting history. ${ }^{22} 245$ These proxy measures probably reflect more a cumulative exposure to repeated subconcussive head impacts rather than concussion per se. Where concussion was self-reported, eight articles included a definition or explanation of how concussion was defined within the study ${ }^{23}{ }^{28-34}$; however, only in six of them ${ }^{28} 2931-34$ was this aligned with the latest Berlin consensus definition. ${ }^{15}$ In two studies, the definition was more compatible with repeated subconcussive head impact. ${ }^{23}{ }^{30}$ Notably, definition of concussion was not provided in two studies which performed an internal comparison ${ }^{2627}$ and approximated by length of career ${ }^{35}$ and number of matches fought (bouts) ${ }^{25}$ in other two. The prevalence of concussion was reported here only for those studies whose sampling frame was considered to be appropriate 272932 (online supplementary table 2 , online supplementary table 3 ).

\section{Outcome ascertainment}

Eight cross-sectional studies reported the prevalence of cognitive impairment, dementia or Alzheimer disease among professional/elite athletes; however, only data coming from the three studies with appropriate sampling were considered here 272932 (see online supplementary table 4). All studies measured the outcome with various screening instruments used for neurocognitive function, five attempted defining a threshold of cognitive impairment using specified cut-offs, that is, the Montreal Cognitive Assessment (MOCA), ${ }^{32}{ }^{36}$ the Modified Telephone Interview for Cognitive Status (TICS-m), ${ }^{27} 37$ multiple cognitive domain scores, ${ }^{24}{ }^{38}$ the AD8 ${ }^{35} 39$ and the Mild Cognitive Impairment Screen. ${ }^{23} 40$ One study used research doctor diagnoses of fixed cognitive deficit, mild cognitive impairment and dementia, ${ }^{30}$ another the self-reported doctor diagnosis of mild cognitive impairment and Alzheimer disease together with spouse reported memory problems ${ }^{29}$ (online supplementary table 3 ). Only five studies attempted a more comprehensive assessment of the cognitive function through a wider battery of tests 2224263132 (online supplementary table 4).

\section{Cognitive function in former elite/professional rugby players} Three studies investigated the cognitive function of retired elite/ professional rugby players in a total of 239 players and 138 in the comparison group from a study conducted in France, ${ }^{27}$ 103 players and 263 in the comparison group from another 


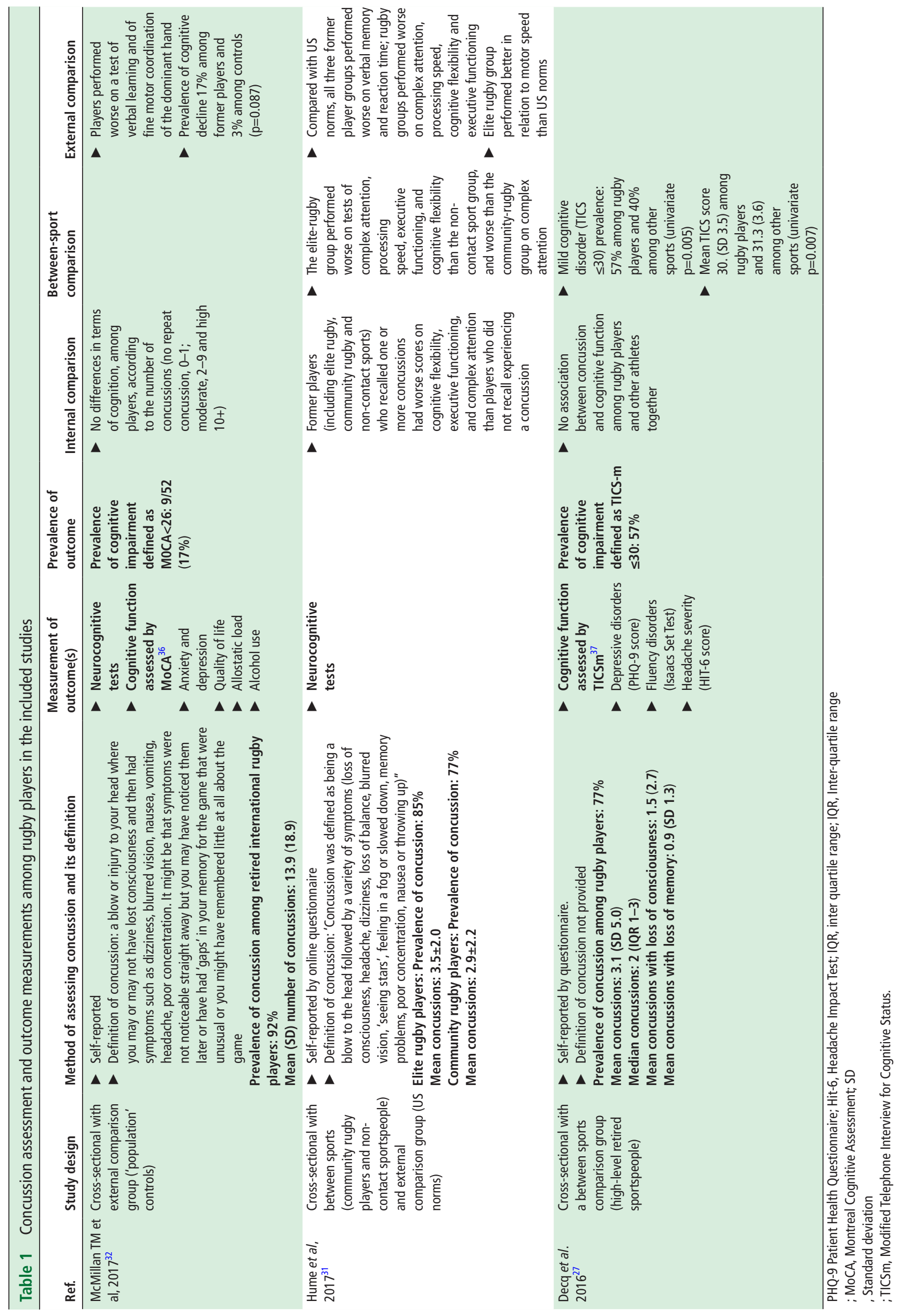




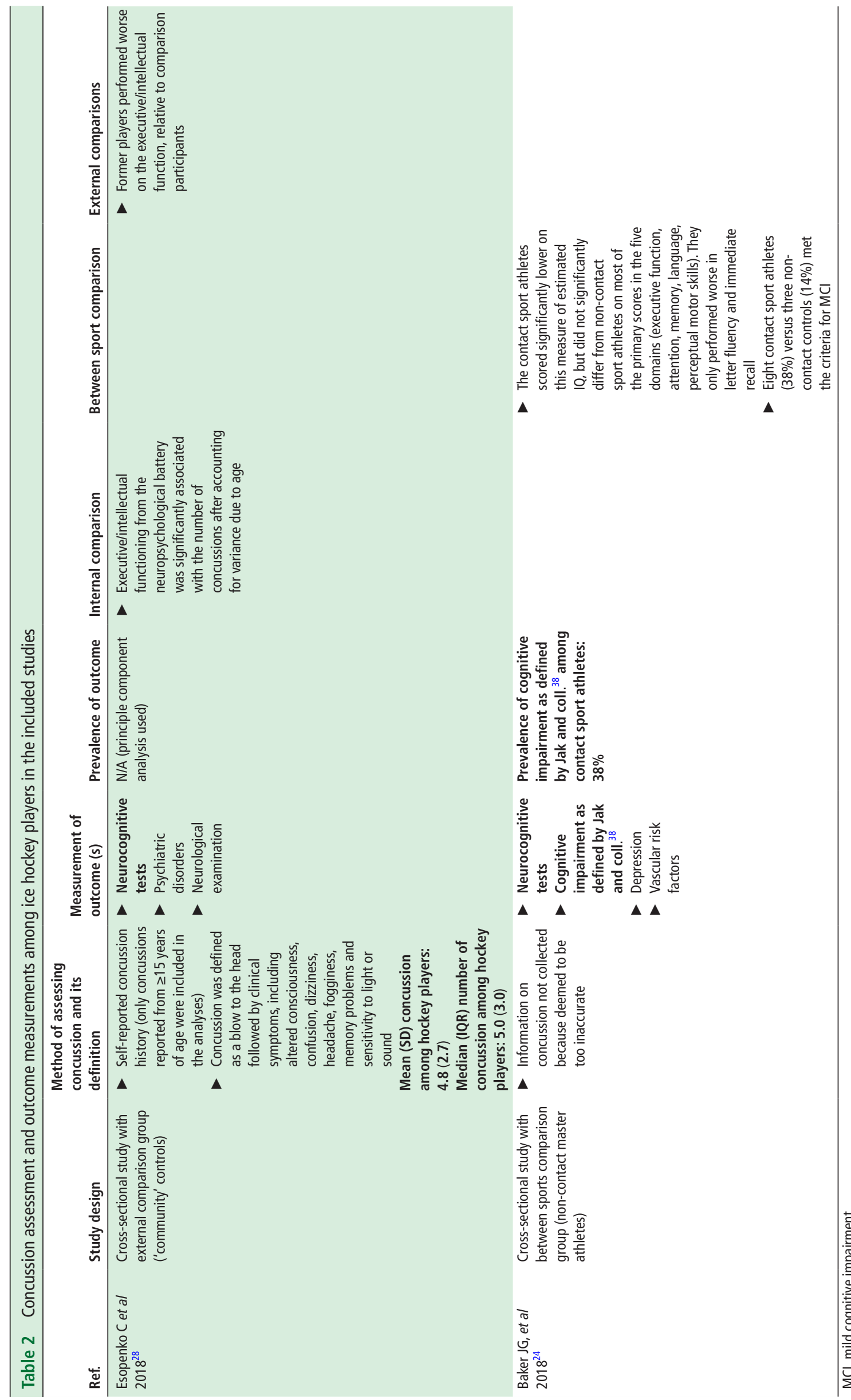




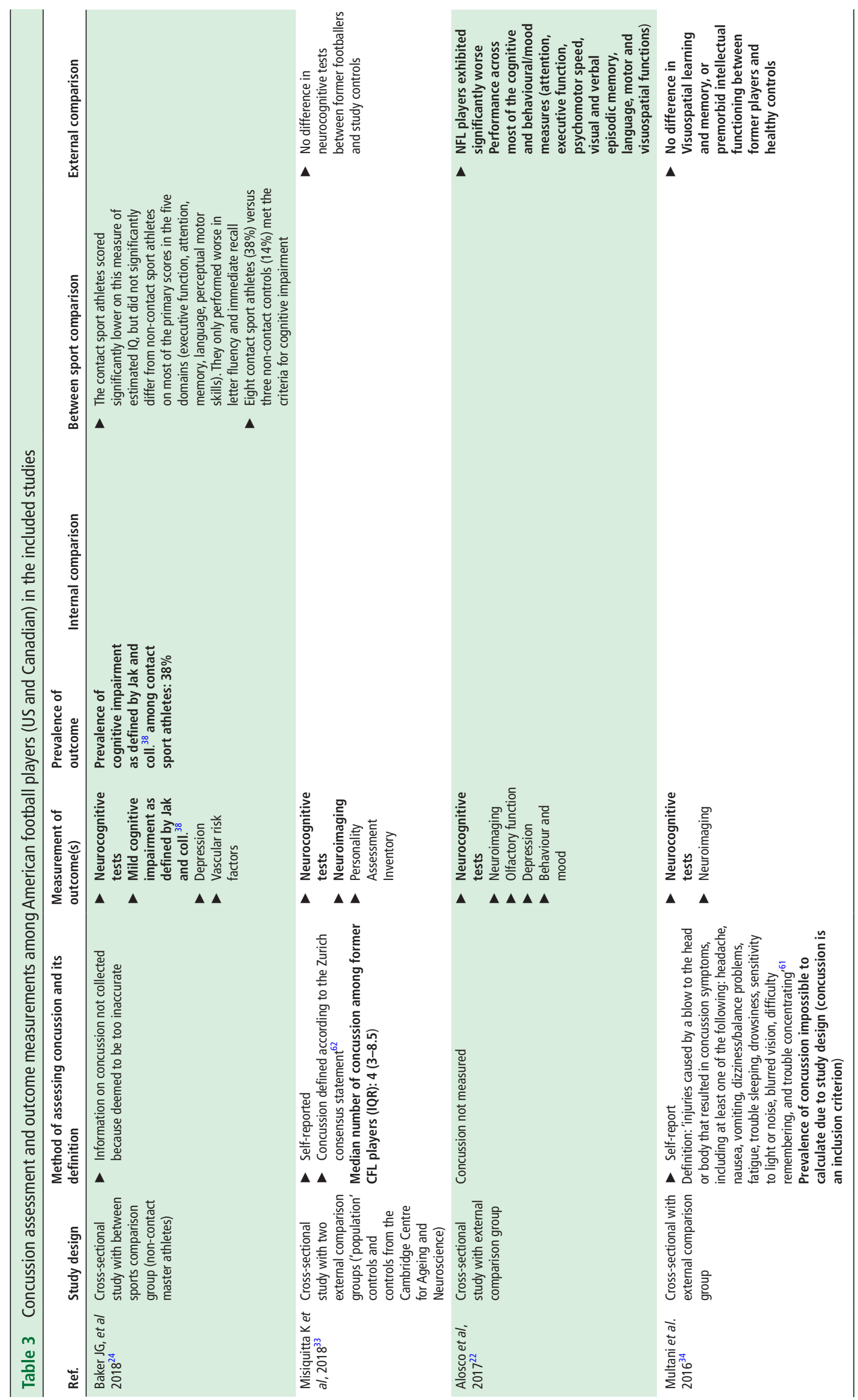

¿ 


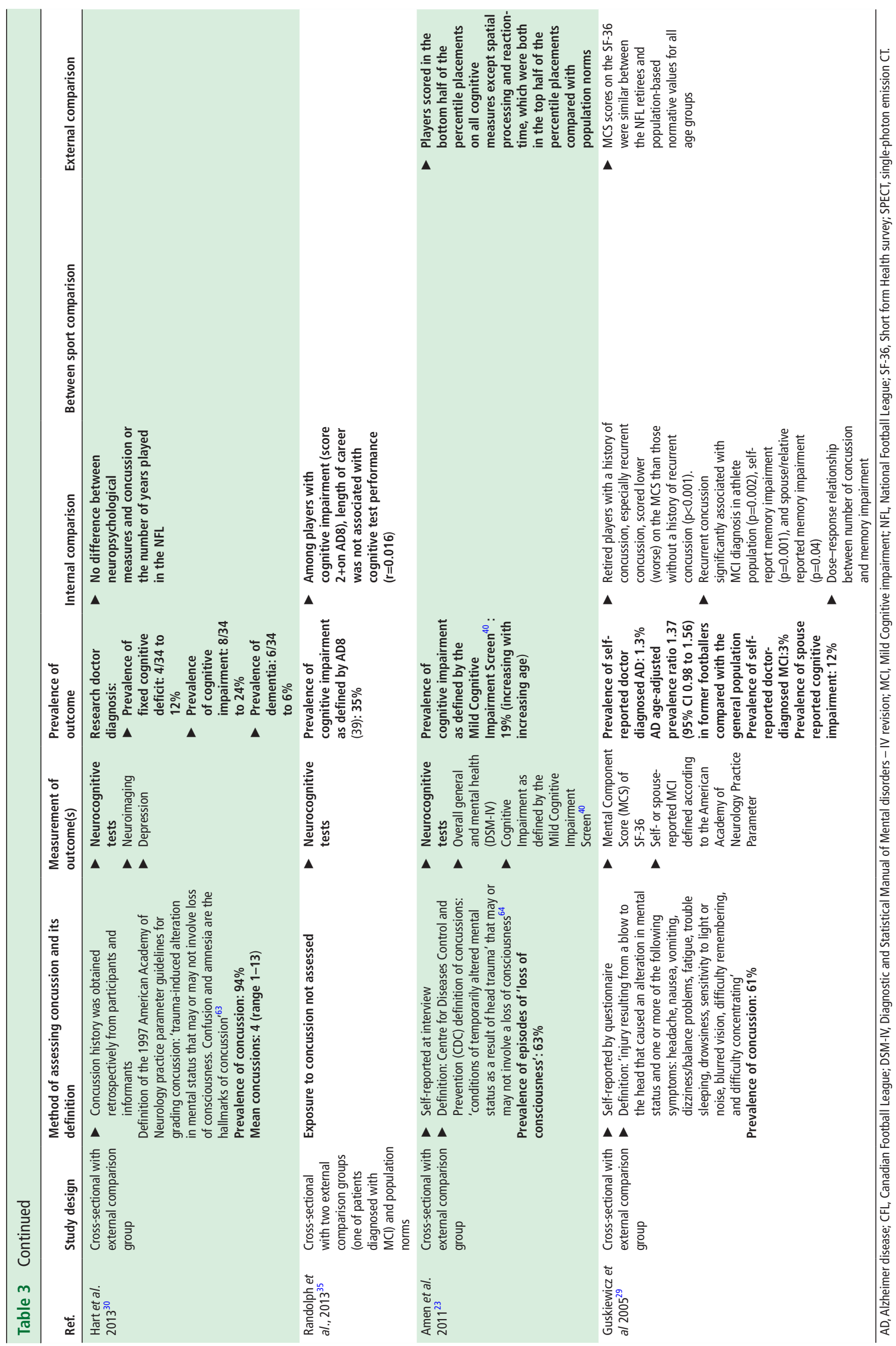




\begin{tabular}{|c|c|c|c|c|c|c|c|}
\hline Ref. & Study design & $\begin{array}{l}\text { Method of assessing } \\
\text { concussion and its } \\
\text { definition }\end{array}$ & $\begin{array}{l}\text { Measurement of } \\
\text { outcome(s) }\end{array}$ & $\begin{array}{l}\text { Prevalence of } \\
\text { outcome }\end{array}$ & Internal comparison & $\begin{array}{l}\text { Between sports } \\
\text { comparison }\end{array}$ & External comparison \\
\hline $\begin{array}{l}\text { Bang et al } \\
2016^{25}\end{array}$ & $\begin{array}{l}\text { Case series with } \\
\text { external comparison } \\
\text { group }\end{array}$ & $\begin{array}{l}\text { Concussion } \\
\text { approximated } \\
\text { by number of } \\
\text { matches fought } \\
\text { (bouts) and } \\
\text { number of KO } \\
\text { Participants had a } \\
\text { mean number of } \\
\text { bout of } 30 \text { (range } \\
23-37 \text { ) and a mean } \\
\text { number of KO of } 1.4 \\
\text { (range } 0-4 \text { ) }\end{array}$ & $\begin{array}{l}\text { Neurocognitive } \\
\text { tests } \\
\text { Neuroimaging } \\
\text { Neurological tests } \\
\text { Personality tests } \\
\text { Mood }\end{array}$ & & - & & $\begin{array}{l}\text { Boxers } \\
\text { performed } \\
\text { significantly } \\
\text { worse in the } \\
\text { delayed recall } \\
\text { of visuospatial } \\
\text { memory } \\
\text { Boxers } \\
\text { performed } \\
\text { significantly } \\
\text { worse in the } \\
\text { assembly task } \\
\text { of the Purdue } \\
\text { Pegboard test } \\
\text { ( } p=0.028 \text { ) but } \\
\text { not in the other } \\
\text { tasks }\end{array}$ \\
\hline $\begin{array}{l}\text { Bernick et al } \\
2015^{42}\end{array}$ & $\begin{array}{l}\text { Cross-sectional study } \\
\text { (baseline of a cohort } \\
\text { study). with external } \\
\text { comparison group }\end{array}$ & $\begin{array}{l}\text { Fight Exposure } \\
\text { Score (FES) } \\
\text { function of } \\
\text { cumulative fights } \\
\quad \text { and intensity of } \\
\quad \text { exposure }{ }^{26} \\
\text { Mean (range) } \\
\text { KO sustained by } \\
\text { professional boxers: } \\
0.9 \text { (0-13) } \\
\text { Mean (range) } \\
\text { KO sustained by } \\
\text { professional Martial } \\
\text { Art Fighters: } 0.6 \\
\text { (0-6) }\end{array}$ & $\begin{array}{l}\text { Neurocognitive } \\
\text { tests } \\
- \text { Neuroimaging }\end{array}$ & & $\begin{array}{l}\text { Reduced } \\
\text { processing } \\
\text { speed in those } \\
\text { exposed to more } \\
\text { fights (p0.041), } \\
\text { worse in boxers } \\
\text { than MMA } \\
\text { Reduced } \\
\text { processing } \\
\text { speed in } \\
\text { those with } \\
\text { a higher FES } \\
\text { with (p=0.023) } \\
\text { with the effect } \\
\text { increasing at } \\
\text { high levels of } \\
\text { FES (however, } \\
\text { the effect was } \\
\text { lost when } \\
\text { restricting to } \\
\text { participants } \\
\text { within an } \\
\text { impaired range }\end{array}$ & & \\
\hline
\end{tabular}

KO, knock-out; MMA, Mixed Martial Arts.

conducted in New Zealand, ${ }^{31}$ and 52 retired players and 46 in the comparison group from one in Scotland. ${ }^{32}$ While the study conducted in France was limited to middle-aged former players with a narrow age range (49-55 years), ${ }^{27}$ the age ranges of the sample of former players in New Zealand and Scotland were much wider (29-72 years in New Zealand; mean age 53.5 (SD 13.0 ) in Scotland); being younger in New Zealand (mean age 41.3 years $($ SD 7.5$))^{3132}$ (table 1 ).

Interpretation of the evidence is hampered by the potential for selection bias. One study was based on participants volunteering to take part, ${ }^{31}$ one study recruited only $15 \%$ of the eligible participants, ${ }^{32}$ and in another study $46 \%$ of former players were invited to participate (corresponding to only $22 \%$ of those initially contacted). ${ }^{27}$

Concussion was self-reported in all studies, and its definition was aligned with the Berlin consensus in two of them. ${ }^{31} 32$ The prevalence of concussion among former rugby players was estimated to range from $77 \%{ }^{27}$ to $92 \% .^{32}$ The mean number of concussions (SD) per player ranged from $3.1(5.0)^{27}$ to 13.9 $(18.9) .^{32}$
More than half of the players evaluated in one study $(57 \%)$ aged 49-55 years were defined as cognitively impaired, ${ }^{37}$ compared with $40 \%$ among the non-contact sport players. ${ }^{27}$ In another, between $2 \%$ and $17 \%$ of former players with a mean age of 53.5 (SD 13.0) were considered cognitively impaired ${ }^{32}$ (table 1).

\section{Internal comparison}

The association between cognitive function and concussion among former rugby players is difficult to assess. The only study reporting this is based on only 52 retired rugby players, divided into no repeated concussion ( 0 to 1 concussion), moderate ${ }^{2-9}$ and high repeated concussions $(10+)$, and no association with cognitive function was shown ${ }^{32}$; this included processing speed, executive function, memory and learning, sustained attention and visual perception. Two of the studies only reported an internal association between concussion and cognitive function among rugby players and other athletes combined. In one of the studies, former elite-rugby players, community-rugby players and non-contact sport players reporting one or more concussions 
had worse scores on cognitive flexibility, executive function and complex attention than players not reporting concussions ${ }^{31}$ (table 1). In another study, concussion was not associated with cognitive function among retired rugby players and high-level sport athletes together in adjusted models ${ }^{27}$ (table 1).

\section{Between-sports comparison}

In one study, the elite-rugby group performed worse on tests of complex attention, processing speed, executive functioning and cognitive flexibility than the non-contact sport group; and worse than the community-rugby group on complex attention. ${ }^{31}$ Additionally, they performed worse than the US norms on verbal memory, reaction time, processing speed, cognitive flexibility and executive functioning. ${ }^{31}$ In another study, overall cognitive function showed median scores lower in retired rugby players than in other sport athletes $(\mathrm{p}=0.007)$, and a higher prevalence of mild cognitive disorders among retired rugby players compared with other sport athletes $(\mathrm{p}=0.005)^{27}$ (table 1).

\section{External comparison}

General cognitive function was not different between 52 retired Scottish rugby players and 46 in the comparison group. Only one former player was considered cognitively impaired; however, using a less conservative cut-off nine former players (17\%) and one comparison group member (3\%) were defined as impaired $(p=0.087){ }^{32}$ When considering single tests, former players exhibited a poorer performance on a test of verbal learning (RAVLT-immediate recall) and on a test of fine motor coordination in the dominant hand (Grooved Pegboard Test), compared with the comparison group. ${ }^{32}$

One of the studies compared the cognitive performance of former elite rugby players and other athletes with US norms. Former rugby players performed worse on processing speed, cognitive flexibility and executive functioning. All athletes (including community rugby players, and cricket and field hockey players) performed worse on verbal memory and reaction time, compared with US norms ${ }^{31}$ (table 1).

\section{Cognitive function in former professional/elite ice hockey players}

Two small studies investigated the association between concussion and cognitive function among former ice hockey players ${ }^{242}$ including a total of 33 and 21 former players with a mean age of $\sim 55$ years $^{24} 28$ (table 2).

Interpretation of results is mainly hampered by the extremely limited sample size in both studies, and by the potential for selection bias: one study recruited former players who volunteered to participate, ${ }^{24}$ in the other, it is not clear how the sample was chosen. $^{28}$

In the only study where information on concussion was collected, its definition was aligned with the Berlin consensus. ${ }^{28}$ The other study deliberately discontinued the collection of data about concussion because the authors deemed the data to be unreliable. $^{24}$

\section{Internal comparison}

Among 33 former ice hockey players, executive/intellectual functioning from the neuropsychological battery was negatively associated with the number of concussions after accounting for $\operatorname{age}^{28}$ (table 2).

\section{Between sports comparison}

A total of 21 contact sport athletes (including American football and ice hockey players combined) scored lower in measures of estimated IQ, but did not differ from non-contact sport athletes on most of the primary scores in the five cognitive domains (executive function, attention, memory, language and perceptual motor skills). However, former players selectively performed worse in letter fluency and immediate recall. ${ }^{24}$ This analysis is largely underpowered to detect even moderately large differences (table 2).

\section{External comparison}

When considering 33 former ice hockey players and 18 members of a comparison group, former players performed worse on executive/intellectual function compared with the comparison group $^{28}$ (table 2).

\section{Cognitive function in former professional/elite American football players}

Eight cross-sectional studies investigated the association between cognitive function and concussion among American football players, ${ }^{23} 24293033-3541$ with samples varying from $758^{29}$ to 18 former players, ${ }^{34}$ with a wide age range (table 3 ).

Interpretation and generalisability of results is potentially hampered by selection bias. One of the two larger studies selected participants on the basis of their cognitive function, and reported information on a follow-up questionnaire with a response rate of $57 \%$ sent out to an initial sample of former players previously recruited with a response rate of $68 \% .^{35}$ The other larger study involved former players likely from the same source (but assessing a different outcome measure), but did not report a response rate, nor gave information on recruitment. ${ }^{29}$ Of two smaller studies, one recruited participants through CTE and Alzheimer disease social media, ${ }^{41}$ the other at former player association meetings and word of mouth. ${ }^{23}$ One of those studies explicitly recruited participants with 'self-reported complaints of cognitive, behavioural and mood symptoms for at least 6 months before study entry', making the differences in cognitive performance when compared with non-concussed and non-symptomatic controls, difficult to interpret. ${ }^{41}$ Recruitment in smaller studies was not clear, ${ }^{24} 33$ apart from one which recruited at former player association meetings, by word of mouth and on a volunteering basis. ${ }^{30}$

Concussion was self-reported in five studies, with a definition aligned with the Berlin consensus in three of them ${ }^{29} 33{ }^{34}$; in the remaining two studies the definition of concussion was potentially overlapping with repeated subconcussive head impacts. ${ }^{23} 30$ The prevalence of concussion among former professional American footballers was estimated to be $61 \%$ among 758 former players $^{29}$; this raised to $63 \%-94 \%$ relaxing the definition criteria to include also repeated subconcussive head impacts ${ }^{23} 30$ (table 3).

The prevalence of poor cognitive function was estimated to be $3 \%$ among 758 former players with a mean age of 53.8 years (SD 13.4) who self-reported symptoms compatible to MCI; however, this raised to $12 \%$ when it was spouse-reported ${ }^{29}$ (table 3 ).

\section{Internal comparison}

The largest, good quality cross-sectional study compared the self-reported or spouse-reported diagnosis of dementia or cognitive impairment among 758 retired professional American footballers. The study performed performing an internal comparison 
among concussed and non-concussed, and then compared the prevalence with estimates from the general population. ${ }^{29}$ The internal comparison found that recurrent concussion was associated with a self-reported doctor-diagnosed cognitive impairment $(p=0.002)$, self-reported memory impairment $(p=0.001)$ and spouse-reported memory impairment $(p=0.04)$ ( $p$ values refer to chi-square tests). ${ }^{29} \mathrm{~A}$ dose-response relationship between number of concussions and cognitive impairment was also found $(\mathrm{p}<0.001)$. Retired players sustaining three or more concussion during their career, have a fivefold prevalence of being diagnosed with MCI and a threefold prevalence of being diagnosed with memory impairment, compared with players with no reported concussion. ${ }^{29}$ Significantly lower scores on the Mental Component Score of the short form health survey (SF-36) were found between concussed (especially recurrently concussed) players and the age-adjusted population norms $(p=0.001) .^{29}$

The other large study screened 513 former American footballers for cognitive function, conducted an analysis of cognitive test results among the 41 found to be cognitively impaired: among them, length of career was not associated with poorer performance. ${ }^{35}$

The only other study reporting an internal comparison was small. Among 34 retired players, no significant correlation between neuropsychological measures and concussion/repeated subconcussive head impact or length of career was found (data not shown) ${ }^{30}$ (table 3 ).

\section{Between-sports comparison}

The only study conducting a comparison with another sport group, compared a total of 21 retired American football and ice hockey players with 21 non-contact sport master athletes, both with age ranges of 36 to 72 years and similar mean age. ${ }^{24}$ Overall, the contact sport athletes scored significantly lower on scores of IQ, letter fluency and immediate memory recall, but did not differ significantly from non-contact athletes in executive function, attention, memory, language and perceptual motor skills (table 3).

\section{External comparison}

The self-reported prevalence of physician-diagnosed Alzheimer diseased among American footballers was estimated to be $1.3 \%$, resulting in an estimated age-adjusted prevalence ratio of 1.37 (95\% C.I. 0.98 to 1.56 ) when compared with the general population. $^{29}$

Five studies conducted an external comparison between former American footballers and samples more or less representative of the general population. In the largest study, involving a sample of 758 retired American footballers, the Mental Component Scale of the SF-36 was similar between the recruited sample and the age-adjusted population-based normative values (although scores were significantly lower when restricted to those concussed). ${ }^{29}$ In an analysis of 128 former players and 28 age-matched volunteers, former players performed worse in the majority of tests assessing attention, executive function, psychomotor speed, visual and verbal episodic memory, language, motor and visuospatial functions, although the significance level did not take into account multiple comparisons. ${ }^{22}$ An analysis of 100 retired American footballers compared with a standardised sample of 810 subjects for the MicroCog test revealed that all players scored in the bottom half of the percentile placements in all measures except spatial processing and reaction-time (both in the top half). ${ }^{23}$

Smaller studies reported no significant differences in neurocognitive tests between former players and the comparison group ${ }^{33} 34$ (table 3).
Cognitive function in former boxers and other fighting sports

Two papers assessed the cognitive function of former boxers: a case series of five professional retired boxers from Korea aged 42-49 years, ${ }^{25}$ and a cross-sectional study with an external comparison group including 93 former boxers and 131 martial art fighters from the US with an age range of 18-44 years ${ }^{26}$ (table 4).

Interpretation of results is hampered by the potential for selection bias, as both studies lack information on sampling or response rate $^{2526}$ (see online supplementary table 2). Concussion per se was not recorded in either of the studies. The larger study on boxers and martial art fighters used a Fight Exposure Score $(\mathrm{FES})^{42}$ to assess the cumulative exposure to concussion as a function of number of professional/elite fights and intensity of exposure. ${ }^{26}$ Exposure to concussion was also measured with number of knock-outs (KOs) sustained. ${ }^{26}$

\section{Internal comparison}

Processing speed among boxers and martial art fighters aged 18-44 years was associated with both number of professional fights $(p=0.041)$, and the FES $(p=0.023)$ with an estimated $0.19 \%$ and $2.1 \%$ reduction in processing speed per fight and unit of FES score increase, respectively. ${ }^{26}$ The proportion of participants impaired in each of the cognitive categories (verbal memory, psychomotor, processing and reaction speed) was calculated for scores below 1.5 the SD of age- and education-matched samples. The proportion of participants with verbal memory and psychomotor speed impairment increased with increasing categories of FES $\left(\mathrm{p}=0.036\right.$ and $\mathrm{p}=0.046$, respectively) ${ }^{26}$ Increasing exposure to concussion (measured either as number of fights or years in professional fighting, or FES) was associated with a decrease in brain structure volumes, particularly of thalamus and caudate $^{26}$ (table 4).

\section{Between sport comparison}

Boxers were shown to have significantly lower scores for processing speed compared with martial art fighters (data not shown $)^{26}$ (table 4).

\section{External comparison}

No significant differences in verbal memory were detected between boxers, fighters and controls after adjustment for age, education and ethnicity. ${ }^{26}$ However, both boxers and martial art fighters showed worse scores of processing speed than the external comparison group, after adjusting for education (data not shown). ${ }^{26}$

In the smaller case series, no significant difference in cognitive function was detected among the five boxers and the four comparison people. However, boxers performed worse on the delayed recall of visuospatial memory compared with the external comparison group ${ }^{25}$ (table 4).

\section{DISCUSSION}

Evidence on the long-term cognitive consequences of concussion experienced in professional/elite sports is accumulating, and overall it suggests the presence of an effect. However, many points to be clarified and dissected remain.

Importantly, the magnitude of the effect is not clear. Studies comparing the prevalence of cognitive impairment and/or dementia among former professional/elite players with different instruments, ${ }^{23} 242729303235$ with other athletes ${ }^{24}$ or other comparison groups, ${ }^{29} 32$ almost invariably ${ }^{29}$ find a difference, with contact sports athletes more affected than the comparison 
group. ${ }^{24} 2732$ However, these differences are very unlikely to be exclusively due to concussion, they could be confounded by any other characteristics of the athletes included (eg, use of licit/illicit drugs, alcohol intake, lifestyle and psychosocial risk factors, etc). Moreover, one would expect to see among the former athletes a healthy cohort effect with decreased prevalence of cardiovascular disease and cancer risk factors, ${ }^{43} 44$ although to what extent this is consistent across generations and across sport disciplines, is to date unclear. ${ }^{45}$ On the other hand, studies investigating cognitive functions with neuropsychological batteries, in most cases find subtle, although statistically significant, differences which are not easy to interpret in terms of clinical significance. ${ }^{28} 29313342$ Small differences on a single test might not reflect a true impairment in that area of functioning of the individual, or may not be noticeable; poor performance on a set of tests does not directly equate to functional disability. ${ }^{46}$ Cognitive test measurements would be more meaningful if they were conducted assessing intra-individual differences (ie, pre-exposure and post-exposure to sport-related concussion(s)), but this would require prospective cohort studies with long follow-up periods which are much more difficult to deliver, and are considerably more expensive and more time consuming than the studies included in this review.

Interestingly, the current evidence summarised in this review is derived by the integration of evidence coming from different comparisons, implying different study designs, but also different inherent risks of bias and errors. It is therefore important to derive and interpret the appropriate conclusion from each comparison under analysis. The internal comparisons, by comparing two groups of people sharing broadly the same characteristics in terms of lifestyle and socioeconomic status, are best positioned to assess the effect of concussion on the outcome, minimising unmeasured and residual confounds. The between-sport comparisons, although aimed at assessing the effect of concussion when comparing contact sports athletes with non-contact sport ones, are also affected by any other systematic difference between sports. For example, dietary supplement and medication use have been shown to be very different among sporting disciplines. ${ }^{47}$ Finally, evidence from the external comparison groups provide grounds for assessing the overall effect of sport participation, including all pros and cons, and it is expected to be associated with better health due to a selection effect, and also partially to the physical activity, and general healthy habits that athletes display in comparison with any non-athlete group (healthy cohort effect), as previously found. ${ }^{43}{ }^{44}$ Nonetheless, this selection effect might be heterogeneous across sports, ${ }^{45}$ and it might be mitigated by the emergence of some lifestyle choices after retirement, as demonstrated for increased body mass index. ${ }^{48}$ However, overall, an inverse associations between participating in sports and general health (specifically for cardiovascular diseases and cancer outcomes) is expected, including the overall risk of dementia when athletes are compared with the general population.

Importantly, the current results refer to professional/elite players only; and it is not clear to what extent this evidence is extendable to varsity or younger pre-professional athletes who might be exposed to an overall lower level of concussion, but might be as or more vulnerable to its consequences. ${ }^{49}$ Unfortunately, the current data do not allow any strong conclusions about potential concussion/cognitive function differences between contact sports. Many sports involve concussion or repetitive low-level head trauma, but it has been argued that each sport should be viewed differently depending on the unique technical and physiological profile that a player is exposed to over the course of a career. ${ }^{5051}$ The ongoing HEalth and Ageing Data IN the Game of football (HEADING) study ${ }^{52}$ will add important information about external and internal comparison of the association between low-impact repetitive head injury and cognitive function among British-based footballers. Recently, Scottish footballers have been shown to be at increased risk of mortality from neurodegenerative diseases, and above all dementia, compared with the general population. ${ }^{53}$

\section{Rugby}

The available evidence indicated an association between sustaining rugby-related concussions and having a worse cognitive function later in life, although to what extent this might be clinically significant, is not entirely clear. None of the reviewed studies produced strong evidence for an effect. The association between concussion and cognitive function (internal comparison) was not detected among Scottish players, although the study was underpowered to detect less than very large effects, ${ }^{32}$ and was small, possibly not very relevant clinically, and at least partially due to multiple comparison in the New Zealand study. ${ }^{31}$ The association was null among French players, although the prevalence of mild cognitive disorders was significantly higher among rugby players compared with other athletes. ${ }^{27}$ This apparent inconsistency might reflect the fact that concussion was not accurately assessed in the study, or might hint to some other systematic differences between these two groups of athletes responsible for the association. The currently ongoing BRAIN study ${ }^{54}$ has used a timeline-assisted interview to increase accuracy of exposure assessment, and it is appropriately powered to detect a difference of 7\% in the Preclinical Alzheimer Cognitive Composite score. ${ }^{5455}$

Nonetheless, the fact that the prevalence of cognitive impairment was estimated to be $17 \%$ among rugby players and $3 \%$ among the comparison group (external comparison), ${ }^{32}$ strongly points towards the presence of an effect, as one would expect former players to be generally healthier compared with the general population, with reduced incidence of noncommunicable diseases (healthy cohort effect), as previously observed. ${ }^{43}{ }^{44}$ However, this external comparison does not allow estimation of the relative impact of concussion or other factors potentially increasing the risk of cognitive impairment among rugby players.

Rugby union only became a professional sport in 1995, and since then the game's dynamics and training has changed substantially increasing the speed of the game, the number of contact events, and the potential for more severe impacts. As a consequence, between-sports comparisons including elite players who played about 30 years ago might underestimate the burden of ill health due to the overall exposure to concussion and other impacts that would apply to current players. On the other hand, the attention to concussion management has increased substantially in the last few years, ${ }^{56}$ leading to measurable health outcomes in other sports. ${ }^{57}$

\section{Ice hockey}

The evidence available for the association between concussion and cognitive impairment among ice hockey players is too sparse to allow any meaningful summary. The only study investigating the association among ice hockey players ${ }^{28}$ provided very limited evidence for an effect of concussions on cognitive health. 


\section{American football}

Despite the greatest number of research papers identified investigating the association between concussion and long-term cognitive function among American Footballers, the quality of reporting does not always allow a thorough assessment of the evidence. Nonetheless, overall the evidence points to an association between increasing number of concussions and poorer cognitive function among American footballers. The strongest evidence comes from the largest, well reported study, which suggested that having sustained a concussion during playing career was associated with worse cognitive function performance with a dose-response effect. ${ }^{29}$ The same result was not replicated when duration of career was used as a proxy measure for exposure to concussion, possibly due to misclassification error. ${ }^{35}$ Importantly, some of the evidence, although not all, ${ }^{34}$ also indicates a possible poorer cognitive function of former players with respect to an external comparison group (external comparison), ${ }^{23}{ }^{41}$ which is consistent with a lack of healthy cohort effect when American footballers were compared with population-based normative values. ${ }^{29}$

Interestingly, a linguistic analysis of interviews of active American football players suggested that exposure to the highimpact sport was associated with an overall decline in language complexity scores over time, suggesting that language complexity decline might be a very early sign to be monitored to predict potential CTE onset. ${ }^{58}$

\section{Boxing}

The evidence on boxing and other fight-based sport relies only on a single research study and a very limited case series. ${ }^{25} 42$ Nonetheless, results are indicative of an association between sustaining KOs or number of fights with poorer cognitive function which is more pronounced for boxers compared with martial art fighters. ${ }^{42}$

\section{Limitations}

The evidence collated in this systematic review does not allow a quantitative summary from a meta-analysis to be derived from the association between sustaining a concussion, or participating in a contact sport, and risk of long-term cognitive function impairment. However, in some studies, the evidence could be indicative of an effect that should be explored in more depth. The main methodological critical points encountered when summarising the available evidence were poor reporting of study methods, evidence coming from non-conventional study designs, and limited adjustment for potential confounders. Embracing more consistently the STROBE ${ }^{59}$ and STROBE-ME ${ }^{60}$ recommendations when reporting epidemiological and molecular epidemiological studies, respectively, would dramatically increase the ability to assess the available evidence and draw meaningful conclusions from existing studies. Most studies reviewed here have opted for a cross-sectional design (with a selection of a more or less representative sample of the sport population) and had an external comparison group, selected with varying methodology. However, in many cases, the comparison group has been chosen explicitly selecting individuals who never sustained a concussion. This increases the potential for differences among the two groups resulting likely in residual and unmeasured confounding. As a consequence, evidence from these studies should be interpreted with caution. Recently, the FIELD study (Football's InfluencE on Lifelong health and Dementia risk) provided strong evidence for an increased mortality from Alzheimer disease and other neurodegenerative diseases among Scottish footballers when compared with the general population. ${ }^{53}$ Evidence for external comparison should be limited to cross-sectional studies with unbiased sample selection. Moreover, cross-sectional studies suffer from recall bias in relation to exposures, and this is particularly important in this setting as the outcome measure is cognitive function, in which early manifestation of impairment is memory problems. In this context, the accuracy of the retrospective assessment of concussion is crucial, and no study validated the tool used for exposure assessment. This is also an additional reason why it is important to contrast the evidence from internal comparisons and between sport comparison, where concussion is assumed to be higher in contact sports compared with non-contact ones. Prospective studies assessing the long-term cognitive and neurological health of current players, thus measuring exposure at the time when it occurs, would be ideally placed to overcome these problems. Moreover, cognitive decline-measured as the difference in measures of cognitive function over time-would be a better way of measuring the outcome, when using measures of cognitive function such as cognitive tests. This would allow for the most refined adjustment for individual variability in terms of intelligence and cognitive function. In addition, a large part of the evidence comes from cognitive function measured using screening instruments such as (TICS-m), ${ }^{27} 37$ brief tests of general cognition (MoCA), ${ }^{32} 36$ or self-report measures (AD-8) ${ }^{35} 39$ with very few reporting a comprehensive neuropsychological battery assessment aimed at assessing multiple domains of cognitive functioning thoroughly. 2431324249

The definition of concussion and the method for its assessment varied greatly across studies, hampering the synthesis of the evidence. While the definition of concussion used in the studies which reported it was aligned with the latest Berlin consensus, ${ }^{15}$ 61-64 there were some differences: some did not explicitly state that loss of consciousness was not required for the definition, and others did not mention that symptoms could appear after a time delay. In addition, studies which approximated the cumulative exposure to concussion with length of career, ${ }^{35}$ or bouts fought ${ }^{25}$ failed to provide a measure enabling comparison a consistent definition of exposure is essential for comparing and synthesising evidence coming from future studies. Of particular interest would be to study the age at first concussion/repeated subconcussive head impact, and the concussion density (ie, number of concussion over a specific period of time) in relation to clinical outcomes. Some evidence suggest that the earlier the impact, the more severe the consequences in terms of cognition. ${ }^{49}$ Moreover, this needs to be clearly differentiated from repeated subconcussive head impacts which do not necessary comply with concussion definition. Better clinical and histopathological definitions of CTE expected from future studies, such as the UNITE (Understanding Neurologic Injury and Traumatic Encephalopathy) study ${ }^{65}$ and the DETECT (Diagnosing and Evaluating Traumatic Encephalopathy using Clinical Tests) Study, will also be essential for conducting appropriate epidemiological studies.

Another caveat preventing the drawing of strong conclusions from the existing evidence is the overall poor adjustment for potential confounders which potentially play a major role, even in internal comparisons. A minimum set of confounders represented by age, sex (when not stratified) and a proxy measure for socioeconomic status and/or education must be considered in all cases when assessing associations with cognitive function. Ideally, also a number of cardiovascular/metabolic risk factors such as hypertension, anthropometry and diabetes should also be taken into consideration, given their strong association with the increased risk of dementia. ${ }^{6-68}$ Previous attempts to summarise 
the available evidence have not consistently taken these methodological characteristic into account, focussing more on the synthesis of results. ${ }^{18}$

Publication bias must be taken into consideration when coming to conclusions from this systematic review. As with many systematic reviews, it could be shaped by the fact that only published results in peer-reviewed journals were considered, and could therefore be excluding research with important and differing results and findings, that could potentially affect deductions made. In addition to this, only publications written in English were included in the review. Unfortunately, the lack of a unique measure of association prevented an analysis using funnel plots or significance tests for publication bias.

In conclusion, high-quality, appropriately designed and powered epidemiological studies are urgently needed to assess the long-term association between sustaining a sport-related concussion (or repetitive subconcussive head impacts) and cognitive impairment later in life.

Twitter Valentina Gallo @drvgallo and Simon PT Kemp @drsimonkemp

Acknowledgements We would like to thank Miss Giulia Seghezzo for proofreading the manuscript .

Contributors Study concept and design: VG; Analysis and interpretation of data: VG, KM, SM, TP and DME; Drafting of the manuscript: VG and KM; Data collection: $\mathrm{KM}, \mathrm{SM}$ and TP; Critical revision of the manuscript for important intellectual content: SK, LJ and NP.

Funding This study was partially funded by the Drake Foundation as part of the BRAIN study funded to London School of Hygiene and Tropical Medicine (EPMSZJ20-10) in collaboration with Queen Mary University of London and the Institute of Occupational Health.

\section{Competing interests None declared.}

Patient consent for publication Not required.

Provenance and peer review Not commissioned; externally peer reviewed.

Open access This is an open access article distributed in accordance with the Creative Commons Attribution Non Commercial (CC BY-NC 4.0) license, which permits others to distribute, remix, adapt, build upon this work non-commercially, and license their derivative works on different terms, provided the original work is properly cited, appropriate credit is given, any changes made indicated, and the use is non-commercial. See: http://creativecommons.org/licenses/by-nc/4.0/.

\section{ORCID iD}

Valentina Gallo http://orcid.org/0000-0002-1268-8629

\section{REFERENCES}

1 Martland HS. Punch drunk. J Am Med Assoc 1928;91:1103-7.

2 Meehan W, Mannix R, Zafonte R, et al. Chronic traumatic encephalopathy and athletes. Neurology 2015;85:1504-11.

3 Corsellis JAN, Bruton CJ, Freeman-Browne D. The aftermath of boxing. Psychol Med 1973;3:270-303.

4 McKee AC, Cantu RC, Nowinski CJ, et al. Chronic traumatic encephalopathy in athletes: progressive tauopathy after repetitive head injury. J Neuropathol Exp Neurol 2009:68:709-35.

5 Fleminger $\mathrm{S}$, Oliver DL, Lovestone $\mathrm{S}$, et al. Head injury as a risk factor for Alzheimer's disease: the evidence 10 years on; a partial replication. J Neurol Neurosurg Psychiatry 2003:74:857-62.

6 Gardner RC, Yaffe K. Epidemiology of mild traumatic brain injury and neurodegenerative disease. Mol Cell Neurosci 2015;66:75-80.

7 Jafari S, Etminan M, Aminzadeh F, et al. Head injury and risk of Parkinson disease: a systematic review and meta-analysis. Mov Disord 2013;28:1222-9.

8 Broglio SP, Eckner JT, Paulson HL, et al. Cognitive decline and aging: the role of concussive and subconcussive impacts. Exerc Sport Sci Rev 2012:40:138-44.

9 Pearce N, Gallo V, McElvenny D. 0497 Head trauma in sport and neurodegenerative disease: an introduction and review of the epidemiological evidence. Occup Environ Med 2017;74:A156.2.

10 Tartaglia MC, Hazrati L-N, Davis KD, et al. Chronic traumatic encephalopathy and other neurodegenerative proteinopathies. Front Hum Neurosci 2014;8:30.

11 Turner RC, Lucke-Wold BP, Robson MJ, et al. Alzheimer's disease and chronic traumatic encephalopathy: Distinct but possibly overlapping disease entities. Brain Injury 2016;30:1279-92.
12 Blennow K, Hardy J, Zetterberg H. The neuropathology and neurobiology of traumatic brain injury. Neuron 2012;76:886-99.

13 Takahata K, Tabuchi H, Mimura M. [Late-onset Neurodegenerative Diseases Following Traumatic Brain Injury: Chronic Traumatic Encephalopathy (CTE) and Alzheimer's Disease Secondary to TBI (AD-TBI)]. Brain Nerve 2016;68:849-57.

14 Mez J, Daneshvar DH, Kiernan PT, et al. Clinicopathological evaluation of chronic traumatic encephalopathy in players of American football. JAMA 2017;318:360-70.

$15 \mathrm{McCrory}$ P, Meeuwisse W, Dvořák J, et al. Consensus statement on concussion in sport-the $5^{\text {th }}$ international conference on concussion in sport held in Berlin, October 2016. Br J Sports Med 2017:51:838-47.

16 Gardner AJ, Howell DR, Levi CR, et al. Evidence of concussion signs in national rugby League match play: a video review and validation study. Sports Med - Open 2017:3:29.

17 Carson A. Concussion, dementia and CTE: are we getting it very wrong? J Neurol Neurosurg Psychiatry 2017:88:462-4.

18 Manley G, Gardner AJ, Schneider KJ, et al. A systematic review of potential long-term effects of sport-related concussion. Br J Sports Med 2017;51:969-77.

19 Moher D, Liberati A, Tetzlaff J, et al. Preferred reporting items for systematic reviews and meta-analyses: the PRISMA statement. Open Med 2009:3:e123-30.

20 Peterson J, Welch V, et al. The Newcastle-Ottawa scale (NOS) for assessing the quality of nonrandomised studies in meta-analyses 2013.

21 Modesti PA, Reboldi G, Cappuccio FP, et al. Panethnic differences in blood pressure in Europe: a systematic review and meta-analysis. PLoS One 2016;11:e0147601.

22 Alosco ML, Jarnagin J, Tripodis Y, et al. Olfactory function and associated clinical correlates in former national football League players. J Neurotrauma 2017:34:772-80.

23 Amen DG, Newberg A, Thatcher R, et al. Impact of playing American professional football on long-term brain function. J Neuropsychiatr 2011:23:98-106.

24 Baker JG, Leddy JJ, Hinds AL, et al. An exploratory study of mild cognitive impairment of retired professional contact sport athletes. J Head Trauma Rehabil 2018;33:E16-23.

25 Bang SA, Song YS, Moon BS, et al. Neuropsychological, Metabolic, and GAB Receptor Studies in Subjects with Repetitive Traumatic Brain Injury. J Neurotrauma 2016;33:1005-14.

26 Bernick C, Banks SJ, Shin W, et al. Repeated head trauma is associated with smaller thalamic volumes and slower processing speed: the Professional Fighters' Brain Health Study. Br J Sports Med 2015;49:1007-11.

27 Decq P, Gault N, Blandeau M, et al. Long-Term consequences of recurrent sports concussion. Acta Neurochir 2016:158:289-300.

28 Esopenko C, Chow TW, Tartaglia MC, et al. Cognitive and psychosocial function in retired professional hockey players. J Neurol Neurosurg Psychiatry 2017;88:512-9.

29 Guskiewicz KM, Marshall SW, Bailes J, et al. Association between recurrent concussion and late-life cognitive impairment in retired professional football players. Neurosurgery 2005;57:719-26. discussion 719-26.

30 Hart J, Kraut MA, Womack KB, et al. Neuroimaging of cognitive dysfunction and depression in aging retired National football League players: a cross-sectional study. JAMA Neurol 2013;70:326-35.

31 Hume PA, Theadom A, Lewis GN, et al. A comparison of cognitive function in former rugby union players compared with former Non-Contact-Sport players and the impact of concussion history. Sports Med 2017:47:1209-20.

32 McMillan TM, McSkimming P, Wainman-Lefley J, et al. Long-Term health outcomes after exposure to repeated concussion in elite level: rugby union players. J Neurol Neurosurg Psychiatry 2017;88:505-11.

33 Misquitta K, Dadar M, Tarazi A, et al. The relationship between brain atrophy and cognitive-behavioural symptoms in retired Canadian football players with multiple concussions. Neuroimage 2018;19:551-8.

34 Multani N, Goswami R, Khodadadi M, et al. The association between white-matter tract abnormalities, and neuropsychiatric and cognitive symptoms in retired professional football players with multiple concussions. J Neurol 2016:263:1332-41.

35 Randolph C, Karantzoulis S, Guskiewicz K. Prevalence and characterization of mild cognitive impairment in retired National football League players. J Int Neuropsychol Soc 2013:19:873-80

36 Nasreddine ZS, Phillips NA, Bédirian V, et al. The Montreal cognitive assessment, MoCA: a brief screening tool for mild cognitive impairment. J Am Geriatr Soc 2005:53:695-9.

37 Cook SE, Marsiske M, McCoy KJM. The use of the modified telephone interview for cognitive status (TICS-M) in the detection of amnestic mild cognitive impairment. J Geriatr Psychiatry Neurol 2009:22:103-9.

38 Jak AJ, Bondi MW, Delano-Wood L, et al. Quantification of five neuropsychological approaches to defining mild cognitive impairment. Am J Geriatr Psychiatry 2009:17:368-75.

39 Galvin JE, Roe CM, Powlishta KK, et al. The AD8: a brief informant interview to detect dementia. Neurology 2005;65:559-64.

40 MedicalCareCorporation. Mild cognitive impairment screen. Irvine, CA, USA, 2007.

41 Alosco ML, Tripodis Y, Fritts NG, et al. Cerebrospinal fluid tau, A $\beta$, and sTREM2 in former national football League players: modeling the relationship between repetitive head impacts, microglial activation, and neurodegeneration. Alzheimer's \& Dementia 2018;14:1159-70 
42 Bernick C, Banks S, Phillips M, et al. Professional fighters brain health study: rationale and methods. Am J Epidemiol 2013;178:280-6.

43 Chio A, Benzi G, Dossena M. Severely increased risk of amyotrophic lateral sclerosis among Italian professional football players. Brain 2005;128:472-6.

44 Lehman EJ, Hein MJ, Baron SL, et al. Neurodegenerative causes of death among retired National football League players. Neurology 2012;79:1970-4.

45 Nguyen VT, Zafonte RD, Chen JT, et al. Mortality among professional American-Style football players and professional American baseball players. JAMA Netw Open 2019;2:e194223.

46 Committee on Psychological Testing IVT, Populations B on the H of S, Medicine I of Cognitive Tests and Performance Validity Tests [Internet]. National Academies Press (US), 2015. https://www.ncbi.nlm.nih.gov/books/NBK305230/

47 Huang S-HS, Johnson K, Pipe AL. The use of dietary supplements and medications by Canadian athletes at the Atlanta and Sydney Olympic Games. Clin J Sport Med 2006;16:27-33.

48 Willeumier K, Taylor DV, Amen DG. Elevated body mass in national football League players linked to cognitive impairment and decreased prefrontal cortex and temporal pole activity. Trans/ Psychiatry 2012;2:e68.

49 Alosco ML, Mez J, Tripodis Y, et al. Age of first exposure to tackle football and chronic traumatic encephalopathy. Ann Neurol 2018;83:886-901.

50 Delaney JS, Al-Kashmiri A, Correa JA. Mechanisms of injury for concussions in University football, ice hockey, and soccer. Clinical Journal of Sport Medicine 2014;24:233-7.

51 Hinton-Bayre AD, Geffen G, Friis P. Presentation and mechanisms of concussion in professional rugby League football. J Sci Med Sport 2004;7:400-4.

52 LSHTM. HEADING study [Internet]. Available: https://www.Ishtm.ac.uk/research/ centres-projects-groups/heading-study [Accessed 20 Sep 2019].

53 Mackay DF, Russell ER, Stewart K, et al. Neurodegenerative disease mortality among former professional soccer players. N Engl J Med 2019;381:1801-8.

54 Gallo V, McElvenny D, Hobbs C, et al. Brain health and healthy ageing in retired rugby union players, the brain study: study protocol for an observational study in the UK. BMJ Open 2017;7:e017990.

55 Donohue MC, Sperling RA, Salmon DP, et al. The preclinical Alzheimer cognitive composite: measuring amyloid-related decline. JAMA Neurol 2014;71:961-70.
56 Chermann JF, Klouche S, Savigny A, et al. Return to rugby after brain concussion: a prospective study in 35 high level rugby players. Asian J Sports Med 2014;5:e24042.

57 McCrea M, Broglio S, McAllister T, et al. Return to play and risk of repeat concussion in collegiate football players: comparative analysis from the NCAA concussion study (1999-2001) and care consortium (2014-2017). Br J Sports Med 2020;54:102-9.

58 Berisha V, Wang S, LaCross A, et al. Longitudinal changes in linguistic complexity among professional football players. Brain Lang 2017;169:57-63.

59 von Elm E, Altman DG, Egger M, et al. Strengthening the reporting of observational studies in epidemiology (STROBE) statement: guidelines for reporting observational studies. BMJ 2007;335:806-8.

60 Gallo V, Egger M, McCormack V, et al. Strengthening the reporting of observational studies in epidemiology - molecular epidemiology (STROBE-ME): an extension of the STROBE statement. PLoS Med 2011:8:e1001117.

61 Guskiewicz KM, McCrea M, Marshall SW, et al. Cumulative effects associated with recurrent concussion in collegiate football players. JAMA 2003:290:2549-55.

62 McCrory P, Meeuwisse WH, Aubry M, et al. Consensus statement on concussion in sport: the 4th International Conference on concussion in sport held in Zurich, November 2012. J Am Coll Surg 2013;216:e55-71.

63 Anon. Practice parameter: the management of concussion in sports (summary statement). Report of the Quality Standards Subcommittee. Neurology 1997; 48:581-5.

64 Sports-Related Recurrent Brain Injuries -- United States [Internet. Available: https:// www.cdc.gov/mmwr/preview/mmwrhtml/00046702.htm [Accessed 20 Sep 2019].

65 Boston University [Internet]. UNITE Study » Alzheimer's Disease Center. Available: https://www.bu.edu/alzresearch/ctecenter/cte-center-research/unite-study/ [Accessed 20 Sep 2019].

66 Elovainio M, Ferrie JE, Singh-Manoux A, et al. Socioeconomic differences in cardiometabolic factors: social causation or health-related selection? Evidence from the Whitehall II cohort study, 1991-2004. Am J Epidemiol 2011;174:779-89.

67 Kivimäki M, Luukkonen R, Batty GD, et al. Body mass index and risk of dementia: analysis of individual-level data from 1.3 million individuals. Alzheimers Dement 2018;14:601-9.

68 Meneilly GS, Tessier DM. Diabetes, dementia and hypoglycemia. Can J Diabetes 2016;40:73-6 